\section{Case-Based Nuclear Medicine}

K.J. Donohoe and A.D. Van den Abbeele, eds.

New York, NY: Thieme Publishers, 2011, 600 pages, $\$ 99.99$

This imaging atlas is a practical, easy-to-read and -understand textbook comprising 166 case studies in nuclear medicine, nuclear cardiology, and PET. This atlas of illustrative case studies will be helpful to radiology and nuclear medicine residents in their daily case interpretations and will serve as a useful and quick review for board examinations for trainees. The cases in each section cover most of the important topics seen in an active nuclear medicine department or private practice. The book is conveniently organized into multiple sections based on organ system imaging in general nuclear medicine, with separate sections devoted to nuclear cardiology, PET, pediatric imaging, and radionuclide therapy. The case studies begin with skeletal scintigraphy and end with pediatric imaging. The number of cases in each section varies, with 31 cases in skeletal imaging, 23 cases in neoplasm imaging, and fewer cases in the other sections. The cardiac, pulmonary, and endocrine imaging sections have 12 case studies each; the vascular section has 9; the biliary, pediatric and inflammation/infection imaging sections have 8 each; the renal and central nervous system sections have 7 each; the neuroendocrine and radionuclide therapy sections have 6 each; and the lymphoscintigraphy section, with 4 cases, is the smallest section.

Each case study begins with a brief clinical indication, followed by technique, image interpretation, and, for most case studies, a differential diagnosis. The final diagnosis and clinical follow-up are then presented, along with a comprehensive discussion of the case, a pearls-and-pitfalls section, and a list of suggested reading. The image interpretation section describes the scan abnormality, and the discussion section gives a brief, comprehensive summary of the relevant organ function as it correlates with the normal and abnormal scan findings along with a pertinent discussion of the disease process in a few short, easily understandable paragraphs. The pearls-and-pitfalls category in every case study briefly reiterates and reinforces the salient points in a few sentences. This book emphasizes the importance of correctly interpreting normal and abnormal scan findings and then correlates the scan findings with the underlying physiology or pathologic process.

Every case study has the same technique repeated almost verbatim. This redundancy is not helpful, as it takes up valuable space and adds pages to the book without adding any useful information to the case discussion. I skipped over each repetition of the same technique after reading the prototype technique in the first case. The imaging technique section is useful for understanding the process of imaging and the interpretation of findings. A practical and more effective approach would be to list the general technique at the beginning of each section and then mention only the special additional technical features used in a particular case. The space saved by avoiding repetition of the technique could be used to enhance the image displays. For example, the renal images in case 115 are suboptimal: the

COPYRIGHT (C) 2012 by the Society of Nuclear Medicine and Molecular Imaging, Inc. small size of images 115.1 (page 387) and 115.5 (page 389) makes it difficult to appreciate the abnormality shown and could be improved if made larger. In case 116, the fine-print text beneath images 116.1 (page 393 ) and 116.4 (page 394) is repetitious and difficult to read and could be improved by being limited to essential details such as the duration of each image and the total imaging time. Case 119, with multiple images on pages 405 and 406, is very busy, with a lot of different images and curves crowded together.

Cases 65 and 66 (pages 200-203) are on parathyroid imaging but are erroneously listed as skeletal scintigraphy rather than endocrine scintigraphy.

The lymphoscintigraphy section briefly mentions applications in breast cancer but includes no images. Because sentinel node localization in breast cancer is one of the most widely used applications of lymphoscintigraphy in most hospitals, future editions would benefit from its inclusion and make the section more complete.

Apart from these few issues, this comprehensive textbook is an excellent compilation of 166 interesting case studies covering most of nuclear and molecular imaging. Although a few of the images can be improved, the image quality overall is adequate to good and the glossy paper enhances the image display. This book showcases most commonly encountered disease processes in nuclear and molecular imaging. The discussions are easy to understand, being concise, clear, and direct. The salient points offered in the pearls-andpitfalls sections reinforce important aspects of each case, making for a useful, comprehensive overview of abnormal scan finding in diverse disease processes. I highly recommend this book to residents in nuclear medicine and radiology and anyone involved in the practice of nuclear medicine. As a quick reference manual to learn from and increase or refresh one's knowledge base, the book will be a valuable addition to any nuclear medicine departmental library.

\section{Usha A. Joseph}

University of Texas Medical School at Houston

6431 Fannin, Suite $2.130 b$

Houston, TX 77030

E-mail: usha.a.joseph@uth.tmc.edu

Published online Aug. 2, 2012.

DOI: 10.2967/jnumed.112.108605

\section{Nanoplatform-Based Molecular Imaging}

X. Chen, ed.

Hoboken, NJ: John Wiley and Sons, Inc., 2011, 848 pages, $\$ 195$

The objectives of this book are to present the rational design of water-soluble, biocompatible nanoparticles for the imaging of cellular functions and their follow-up. Also, molecular imaging probes based on nanotechnology are presented, along with their potential applications for diagnosis, intervention, and response to 